\title{
Proyecto HU-CI: una necesidad en tiempos de la COVID-19
}

\author{
HU-CI Project: A necessity in times of COVID-19 \\ José de Jesús Bohórquez-Rivero iD ${ }^{1}$, Angélica Patricia \\ Montenegro-Gómez iD ${ }^{1}$, José Gabriel Restom-Arrieta (D)1, José David \\ Sáenz-López $\mathbb{D D}^{1}$ y Emilio Abuabara-Franco $\mathbb{D}^{2}$

\footnotetext{
${ }^{1}$ Grupo de Investigación Gibacus. Escuela de Medicina. Universidad del Sinú, Seccional Cartagena. Cartagena de Indias, Colombia.
} \\ ${ }^{2}$ Departamento de Medicina Interna. Universidad del Sinú, Seccional Cartagena. Cartagena de Indias, Colombia.
}

Cómo citar: Bohórquez-Rivero JJ, Montenegro-Gómez AP, Restom-Arrieta JG, Sáenz-López JD, Abuabara-Franco E. Proyecto HU-CI: una necesidad en tiempos de la COVID-19. Rev. Colomb. Nefrol. 2021; 9(1), e509. https://doi.org/10.22265/acnef.9.1.509

Recibido: 19/Ago/2020

Aceptado: 12/Mar/2021

Publicado: 14/Dic/2021

\section{Estimado editor:}

A lo largo de la historia nos hemos visto sometidos a diversas situaciones en las cuales se ha evidenciado cuan vulnerable somos. La nueva enfermedad por coronavirus 2019 (COVID- 19), ha logrado propagarse rápidamente a la mayoría de los países, y ha generado elevadas tasas de morbimortalidad. De igual manera, ha logrado perturbar la mayoría de esferas cotidianas de las personas, y ha colapsado la mayor parte de los sistemas de salud, la economía y demás actividades a nivel mundial.

Las unidades de cuidado intensivo (UCI) juegan un papel clave en este momento, debido a que una gran cantidad de pacientes con COVID-19 progresan a síndrome de dificultad respiratoria aguda (SDRA), sepsis y shock, requiriendo estancia y tratamiento en dicho lugar.

$\square$ Correspondencia: Universidad del Sinú, Seccional Cartagena, Tv. 54 \#41-117, Cartagena de Indias, Colombia. Correo electrónico: josedsaenzlopez@hotmail.com 
En este contexto parece adecuado realizarnos la siguiente pregunta: ¿Qué estamos haciendo en las UCI de Colombia en medio de esta emergencia sanitaria por la COVID-19? Responder a esta reflexión nos abre una ventana para trabajar en la humanización de las unidades de cuidados intensivos del país. Sin duda, los avances científicos de los últimos años han ayudado a disminuir la mortalidad en los pacientes, pero también han conseguido que el personal de la salud se aleje sutilmente del contacto humano; es decir, que se centren en la patología y se olviden del ser humano que padece la patología [1]. Para abordar este tema debemos aclarar el concepto de "humanizar", el cual es definido por la Real Academia Española como "Hacer humano, familiar y afable a alguien o algo" [2].

La humanización de la asistencia médica consiste en llenar de valores propiamente humanos la atención en salud, sin fundamentar el pilar de tratamiento únicamente en la ciencia y la técnica; de esta manera se lograría aumentar los valores humanos de los profesionales de la salud hacia las necesidades de los pacientes y sus familiares [2,3].

Como gran referente del tema nos permitimos citar al denominado Proyecto HU-CI, el cual nació en febrero del 2014 en la UCI del Hospital Universitario de Torrejón, en Madrid, entre cuyos propósitos se incluyen la flexibilización de los horarios de visita, comunicación, bienestar del paciente, presencia y participación de la familia, cuidado al profesional, prevención, manejo y seguimiento del síndrome poscuidados intensivos, arquitectura humanizada y cuidados al final de la vida [4].

Dicho proyecto aporta grandes beneficios para todos los que interactúan en él. Para el paciente crítico es de gran ayuda al aminorar el malestar, dado que varios estudios han demostrado que el dolor, la ansiedad y separación familiar son algunas de las causas del sufrimiento en esta población [5], situación que sin duda empeora su cuadro clínico. Está en las manos del equipo encargado, trabajar estos aspectos, instaurando guías de manejo, promoviendo palear dolores, evitando la interrupción del sueño, el delirio, la agitación, la depresión y la inmovilidad. La implementación de estas medidas no solo reduce la mortalidad, sino que disminuye la estancia media o el tiempo de ventilación mecánica al que es sometido el paciente y aminora secuelas psicológicas, cognitivas y físicas en los pacientes que se encuentran en estos servicios [6]. En otras palabras, al ejecutar este proyecto en Colombia se espera disminuir el síndrome poscuidados intensivos que afecta a un número importante de pacientes y más en estos tiempos de la COVID-19, donde las UCI se encuentran colapsadas $(30 \%$ a $50 \%)[4]$. 
Otro pilar fundamental en la obra de este proceso es el familiar; los familiares son indispensables en el acompañamiento de los pacientes y su satisfacción tiene un valor extra. Se les debe explicar adecuadamente la condición del paciente, se les aclare en su totalidad las dudas respecto a diagnóstico y pronóstico, dado que en muchas ocasiones estos no entienden claramente los procesos de enfermedad que afronta su familiar, por lo que debe ser desglosado minuciosamente para su total comprensión [7]. Se les debe brindar también apoyo emocional y/o religioso en medio de la calamidad familiar que afrontan a través de la colaboración de psicólogos, religiosos y/o trabajadores sociales; esto ayudaría a disminuir las secuelas psicológicas de los familiares y acompañantes. En este grupo se ha demostrado que frecuentemente sufren de ansiedad (70\%), depresión (35\%) y estrés postraumático (35\%), lo que en algunos casos suele persistir durante meses posteriores al alta del familiar o ser querido [4]. Situación que disminuiría significativamente al ejecutar el proyecto HU-CI.

El tercer eslabón de esta cadena lo constituyen los profesionales que trabajan en las UCI, personal sanitario como: médicos, enfermeros, auxiliares, psicólogos, entre otros, y personal no sanitario, donde se incluyen arquitectos informáticos, diseñadores, profesores, administrativos y demás. Esto, considerando que el ideal de humanizar las UCI no solo comprende el trato del personal sanitario con pacientes y familiares, sino mejorar todo el entorno, buscando que sea más agradable para aquellos que interactúan en él [2]. Es decir, en esta iniciativa se reconoce a todo aquel que interactúa en aras de hacer humano el servicio prestado y no excluye de esto al personal sanitario, gremio que en ocasiones puede llegar a ser juzgado por los familiares de los pacientes; por lo tanto, al instaurar este proyecto se hace sumamente importante entender y aceptar la falibilidad y vulnerabilidad de los seres humanos, y que estos gozan del derecho a demostrar sanamente sus emociones; razón por la cual, incluir ayuda emocional a los profesionales sanitarios y no sanitarios es esencial.

Sin lugar a dudas, el dolor de aquellos que están al cuidado del personal de la salud también afecta emociones de este gremio. En la integralidad de este cambio se deben afrontar con respeto todas estas situaciones y complementar con todos los recursos que estén a disposición para hacer real esta propuesta, incluyendo capacitaciones que brinden herramientas para la gestión de emociones y las inseguridades que en momentos puede provocar el dolor ajeno [6]. Al abrirse a un trato más humano es necesario tomar conciencia de que el compromiso inicialmente es voluntad propia a mejorar el servicio brindado, mejorar la calidad de las relaciones y del entorno. El hecho de humanizar tiene la necesidad de no plantarse únicamente en lo que se cree que es necesario para el paciente, sino abrirse al diálogo con este y sus familiares, para escuchar sus necesidades individuales. La actitud y el ánimo que sea posible brindar al paciente representan una herramienta fundamental para 
la adherencia al cambio y, en consecuencia, contribuir de manera eficiente a la mejora de la condición del paciente [2].

No es un camino fácil, pues ningún cambio drástico lo es. Disponer de mayor cooperación por parte de todos los involucrados es una decisión que se asume desde el compromiso a trabajar en un sano ambiente con los demás, lo que ayuda a prestar un servicio de calidad ideal. Diseñar estrategias adaptadas a los requerimientos humanos es fundamental; desafortunadamente la crisis actual por la COVID-19 ha revelado imperativamente esta necesidad, que se debió haber pensado tiempo atrás. Sin embargo, aún no es tarde, es el momento de tomar esta coyuntura como un llamado a la conciencia y optimización del trabajo en esta área. En este sentido, en el marco de la actual pandemia, sin duda la instauración del proyecto HU-CI es una pieza fundamental que acoge la importancia de conocer la necesidad de los enfermos y distinguir que no solo se trata una enfermedad, sino que se trata de un ser humano que tiene emociones y familia que vela por su recuperación.

Teniendo en cuenta lo previamente dicho, consideramos que se debe ampliar la investigación en dicho tópico, enfatizando en aquellas problemáticas que podemos solucionar prontamente desde el diálogo, tanto en el servicio hospitalario como con los familiares de los pacientes. Se deben aprovechar también los avances tecnológicos y de programación para integrarlos a este proyecto, con el fin de facilitar la comunicación de todos los sectores, promoviendo las relaciones humanas y de comunicación entre familiar-paciente, personal sanitario-familiar, paciente-personal sanitario y personal de apoyo para mejorar la condición emocional de todos los involucrados.

Por último, hacemos un respetuoso llamado a la reflexión con la siguiente pregunta: ¿Por qué no ser parte de este gran cambio?, tomar la decisión de ser más humanos en el trato con quienes nos rodean sería provechoso para todos. Incluso el mismo sistema de salud se verá beneficiado, teniendo en cuenta que las estrategias del proyecto $\mathrm{HU}-\mathrm{CI}$ ayudan a disminuir la estancia hospitalaria y las consecuencias derivadas del ingreso en UCI, como el síndrome posUCI; lo que directamente se vería reflejado en la disminución del gasto económico, no solo en las UCI, sino en su posterior reinserción a la vida funcional, logrando una mayor satisfacción para los pacientes y sus familiares. La incorporación de este proyecto es fundamental en estos tiempos difíciles y adhiriéndonos a sus líneas estratégicas estaríamos trazando el camino hacia el futuro de una medicina cada vez más centrada en las personas. La pandemia no nos lo va a poner fácil, ya que ha alterado los tres pilares de cualquier sistema de salud (pacientes, familiares y profesionales), con la sobrecarga a todos los niveles que nos está suponiendo, pero todo es cuestión de perseverancia. 


\section{Conflicto de intereses}

Ninguno que declarar.

\section{Financiación}

No se recibió financiación.

\section{Referencias}

[1] La Calle GH, Lallemand CZ. HUCI se escribe con H de HUMANO. Enferm Intensiva. 2014;25(4):123-124. DOI: https://doi.org/10.1016/j.enfi.2014.11.001 $\uparrow$ Ver página 2

[2] March JC. Humanizar la sanidad para mejorar la calidad de sus servicios. Rev Calid Asist. 2017;32(5):245-247. DOI: https:/doi.org/10.1016/j.cali.2017.10.001 个Ver página 2, 3, 4

[3] Holanda Peña MS, Talledo NM, Ots Ruiz E, Lanza Gómez JM, Ruiz Ruiz A, García Miguelez A, et al. Satisfacción en la Unidad de Cuidados Intensivos (UCI): la opinión del paciente como piedra angular. Med Intensiva. 2017;41(2):78-85. DOI: https://doi.org/10.1016/j.medin. 2016.06.007 $\uparrow$ Ver página 2

[4] Martín Delgado MC, García de Lorenzo y Mateos A. Sobrevivir a las unidades de cuidados intensivos mirando a través de los ojos de la familia. Med Intensiva. 2017;41(8):451-453. DOI: https://oi.org/10.1016/j.medin.2017.02.003 个Ver página 2, 3

[5] Lombardo V, Vinatier I, Baillot ML, Franja V, Bourgeon-Ghittori I, Dray S, et al. How caregivers view patient comfort and what they do to improve it: a French survey. Ann Intensive Care. 2013;3(1):19. DOI: https://doi.org/10.1186/2110-5820-3-19个Ver página 2

[6] Alonso Ovies Á, Heras la Calle G. Humanizar los cuidados reduce la mortalidad en el enfermo crítico. Med Intensiva. 2020;44(2):122-124. DOI: https://doi.org/10.1016/j.medin.2019. $03.002 \uparrow$ Ver página 2, 3

[7] Rodríguez Huerta MD, Álvarez Pol M, Fernández Catalán ML, Fernández Vadillo R, Martín Rodríguez M, Quicios Dorado B, et al. An informative nursing intervention for families of patients admitted to the intensive care unit regarding the satisfaction of their needs: The INFOUCI study. Intensive Crit Care Nurs. 2019;55:102755. DOI: https://doi.org/10.1016/ j.iccn.2019.102755 个Ver página 3 